\title{
PHASE PORTRAITS OF PIECEWISE LINEAR CONTINUOUS DIFFERENTIAL SYSTEMS WITH TWO ZONES SEPARATED BY A STRAIGHT LINE
}

\author{
SHIMIN LI ${ }^{1}$ AND JAUME LLIBRE ${ }^{2}$
}

\begin{abstract}
This paper provides the classification of the phase portraits in the Poincaré disc of all piecewise linear continuous slow-fast differential systems with two zones separated by a straight line. The sufficient and necessary conditions for existence and uniqueness of limit cycles are also given.
\end{abstract}

\section{INTRODUCTION AND STATEMENT OF THE MAIN RESUlts}

The Liénard second order differential equation

$$
\ddot{x}+f(x) \dot{x}+g(x)=0,
$$

where the dot denotes differentiation with respect to the time $t$ and $f, g \in C^{1}$ was first introduced by Liénard in [20]. This differential equation has many important applications in several branches of science, such as biology, chemistry, mechanics, electronics and so on.

The Liénard second order differential equation (1) can be written as the equivalent planar differential system

$$
\begin{aligned}
& \frac{\mathrm{d} x}{\mathrm{~d} t}=y-F(x), \\
& \frac{\mathrm{d} y}{\mathrm{~d} t}=g(x),
\end{aligned}
$$

where $F(x)=\int_{0}^{x} f(t) d t$.

When $F(x)$ and $g(x)$ are polynomial functions in the variable $x$, the Liénard differential system (2) is called the generalized polynomial Liénard differential system has been studied extensively, see $[14,38,41]$ for center conditions, $[13,16,21$, $22,27,37]$ for the number of limit cycles, $[1,40]$ for the amplitude of limit cycles, $[15,26]$ for integrability conditions, $[38,39]$ for isochronous conditions, and $[2,3,17]$ for global phase portraits and bifurcation diagrams.

The investigation of differential system (2) are mainly extended into two directions. On one hand, piecewise linear differential systems are the most natural

2010 Mathematics Subject Classification. 34C37, 34C07, 37G15.

Key words and phrases. Phase portraits, Piecewise linear slow-fast differential system, Limit cycle. 
extensions to the nonlinear differential systems in order to capture nonlinear phenomena observed in real world applications. There are several papers studying the piecewise linear continuous Liénard systems, see [23, 24, 25, 32] and references therein. On the other hand, differential systems with multiple time scales, especially for slow-fast systems, regularly appear in a great variety of areas. Discussions regarding Liénard type slow-fast systems have dominated research in recent years, see $[4,9,18,19,30,31,36]$ for smooth cases, and $[5,6,12,35]$ for piecewise smooth cases.

Motivated by the above two lines of research, the aim of this paper is to investigate the global dynamics of the planar piecewise linear continuous slow-fast differential systems of the form

$$
\begin{aligned}
& \frac{\mathrm{d} x}{\mathrm{~d} t}=y-f(x), \\
& \frac{\mathrm{d} y}{\mathrm{~d} t}=\varepsilon(a-x),
\end{aligned}
$$

where $f(x)$ is a continuous piecewise linear function given by

$$
f(x)= \begin{cases}k_{1} x & \text { if } x \geqslant 0, \\ k_{2} x & \text { if } x \leqslant 0,\end{cases}
$$

with $\varepsilon>0$ sufficiently small and $k_{1} k_{2} \neq 0$.

System (3) is the most simplicity piecewise linear continuous slow-fast differential system and has been studied in the papers [7, 33, 34]. From the results of [33], we can conclude that if $k_{1}=-k_{2}$, then system (3) has neither maximal nor faux maximal canard orbits exist. In 2016 Roberts [34] proved that if $k_{1}>0$ and $k_{2}<0$, then system (3) can have an unstable canard limit cycle. He also investigated the mechanism of canard explosive. If $k_{1}=k_{2}$, then system (3) becomes a smooth linear slow-fast system. In 2006 Dumortier [7] studied the behaviour near infinity on the Poincaré disc.

For the piecewise linear continuous differential systems (3) it is known (see for instance the Chapter 1 of [8]) that the separatrices include all the infinite singular points, all the finite singular points, the separatrices of the hyperbolic sectors of the finite and infinite singular points, and the limit cycles. If $\Sigma$ denote the set of all separatrices in the Poincaré disc $\mathbb{D}^{2}, \Sigma$ is a closed set and the component of $\mathbb{D}^{2} \backslash \Sigma$ are called the canonical regions. See subsection 2.1 for the definition of the Poincaré compactification, there it is defined the Poincaré disc $\mathbb{D}^{2}=\left\{\left(s_{1}, s_{2}\right)\right.$ : $\left.s_{1}^{2}+s_{2}^{2} \leqslant 1\right\}$. We denote by $\mathrm{S}$ and $\mathrm{R}$ the number of separatrices and canonical regions, respectively.

We say that two phase portraits of $X_{1}$ and $X_{2}$ of systems (3) are topologically equivalent if there exists a homeomorphism $h: \mathbb{D}^{2} \rightarrow \mathbb{D}^{2}$ such that $h\left(\partial \mathbb{D}^{2}\right)=\partial \mathbb{D}^{2}$, $h\left(\mathbb{D}^{2} \cap\left\{s_{1}=0\right\}\right)=\mathbb{D}^{2} \cap\left\{s_{1}=0\right\}$, either $h\left(\mathbb{D}^{2} \cap\left\{s_{1}>0\right\}\right)=\mathbb{D}^{2} \cap\left\{s_{1}>0\right\}$ and $h\left(\mathbb{D}^{2} \cap\left\{s_{1}<0\right\}\right)=\mathbb{D}^{2} \cap\left\{s_{1}<0\right\}$, or $h\left(\mathbb{D}^{2} \cap\left\{s_{1}>0\right\}\right)=\mathbb{D}^{2} \cap\left\{s_{1}<0\right\}$ and $h\left(\mathbb{D}^{2} \cap\left\{s_{1}<0\right\}\right)=\mathbb{D}^{2} \cap\left\{s_{1}>0\right\}$, and $h$ mapping orbits of $X_{1}$ into orbits of $X_{2}$ either preserving the orientation, or reversing the orientation of all orbits.

We denote

$$
\Delta_{1}=k_{1}^{2}-4 \varepsilon, \quad \Delta_{2}=k_{2}^{2}-4 \varepsilon
$$


According to the sign of $a, \Delta_{1}, \Delta_{2}$, we classified all the global phase portraits of system (3) with $k_{1} k_{2} \neq 0$. Without loss of generality we assume that $k_{2}>0$. Otherwise we can do the change $X=x, Y=-y, T=-t$, and then obtain $k_{2}>0$. Our main results are the following two theorems.

Theorem 1. The phase portrait on the Poincaré disc of piecewise linear continuous differential system (3) with $k_{1}>0, k_{2}>0$ and $\varepsilon>0$ sufficiently small, or the phase portrait with the sense of all orbits reversed, is topologically equivalent to one of the 15 phase portraits described in Figure 1.

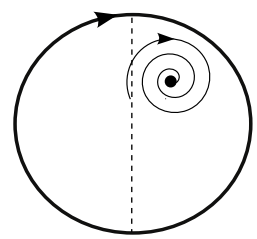

$1.1 S=2, R=1$

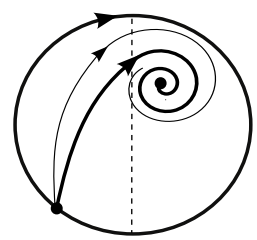

$1.4 S=4, R=1$

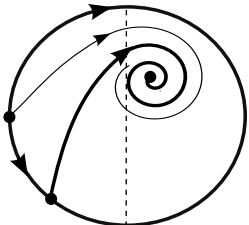

$1.7 S=6, R=1$

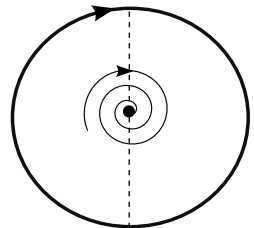

$1.10 S=2, R=1$

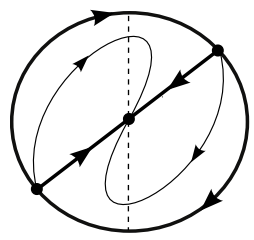

$1.13 S=7, R=2$

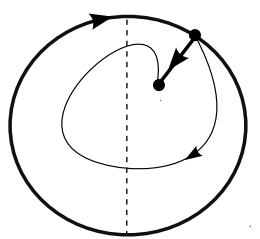

$1.2 S=4, R=1$

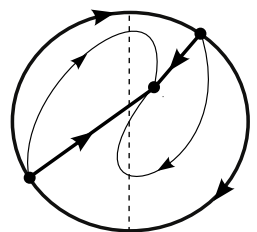

$1.5 S=7, R=2$

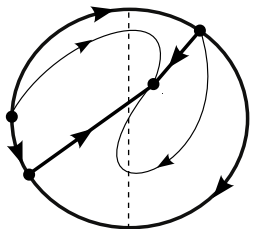

$1.8 S=9, R=2$

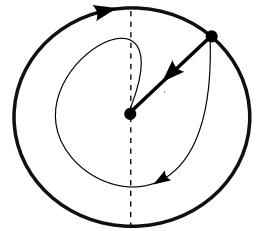

$1.11 S=4, R=1$

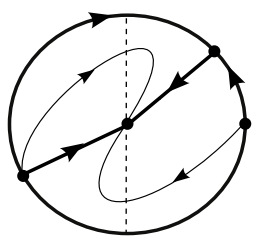

$1.14 S=9, R=2$

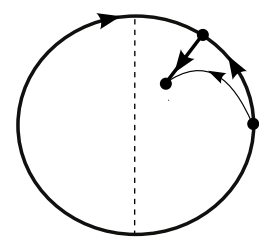

$1.3 S=6, R=1$

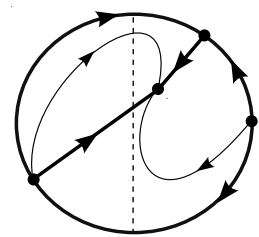

$1.6 S=9, R=2$

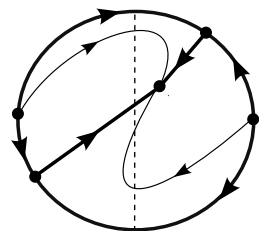

$1.9 S=11, R=2$

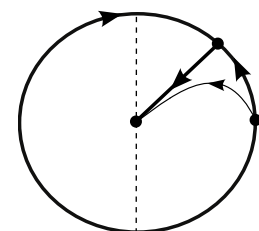

$1.12 S=6, R=1$

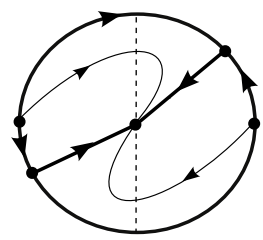

$1.15 S=11, R=2$

Figure 1. Topological phase portraits of system (3) with $k_{1}>$ $0, k_{2}>0$ in Theorem 1 . The dashed line represents the straight line of $x=0$. 
Theorem 2. The phase portrait on the Poincaré disc of piecewise linear continuous differential system (3) with $k_{1}<0, k_{2}>0$ and $\varepsilon$ sufficiently small, or the phase portrait with the sense of all orbits reversed, is topologically equivalent to one of the 18 phase portraits described in Figure 2.

From Figures 1 and 2, we know that system (3) has at most one limit cycle, this result was conjectured by Lum and Chua [28, 29] in 1990, and proved in 1998 by Freire et al [11]. Later on a new and shorter proof was given by Llibre et al in [23]. The following corollary outlines the conditions for the existence and stability of limit cycle. The result on the stability is new.

Corollary 3. Piecewise linear continuous slow-fast differential system (3) with $k_{1}<0, k_{2}>0$ has a unique limit cycle if and only if one of the following six conditions hold:

(I) If $a>0, \Delta_{1}<0, \Delta_{2}<0$ and $k_{1}+k_{2}>0$, then the limit cycle is stable, see Figure2.1.

(II) If $a>0, \Delta_{1}<0, \Delta_{2}=0$, then the limit cycle is stable, see Figure2.5.

(III) If $a>0, \Delta_{1}<0, \Delta_{2}>0$, then the limit cycle is stable, see Figure2.8.

(IV) If $a<0, \Delta_{1}<0, \Delta_{2}<0$ and $k_{1}+k_{2}<0$, then the limit cycle is unstable, see Figure2.1 for reversing all the orientation of the orbits.

(V) If $a<0, \Delta_{1}=0, \Delta_{2}<0$, then the limit cycle is unstable, see Figure 2.5 for reversing all the orientation of the orbits.

(VI) If $a<0, \Delta_{1}>0, \Delta_{2}<0$, then the limit cycle is unstable, see Figure2.8 for reversing all the orientation of the orbits.

Note that statements (I) and (IV) of Corollary 3 can be obtained from Proposition 4 and Theorem 5 of the paper [10].

This paper is organized as follows. In section 2, we first introduce the Poincaré compacification, and then analyze the local phase portraits of the finite and infinite singular points. Applying the results from section 2 we prove our main theorems in section 3. Finally we discuss the differences of phase portraits between smooth and piecewise smooth differential systems in section 4 .

\section{Singular POints}

In order to analyze the global behaviour of trajectories it is possible to use Poincaré compactification, see for example the chapter 5 of the book [8].

2.1. Poincaré compactification. Let $\mathbb{S}^{2}$ be the set of points $\mathbf{s}=\left(s_{1}, s_{2}, s_{3}\right) \in \mathbb{R}^{3}$ such that $s_{1}^{2}+s_{2}^{2}+s_{3}^{2}=1$. We will call this set the Poincaré sphere. Given a polynomial differential system

$$
\begin{aligned}
& \dot{x_{1}}=P\left(x_{1}, x_{2}\right), \\
& \dot{x_{2}}=Q\left(x_{1}, x_{2}\right),
\end{aligned}
$$

of degree $d=\max \{\operatorname{deg}(P), \operatorname{deg}(Q)\}$ in $\mathbb{R}^{2}$ identified with the plane $x_{3}=1$ of $\mathbb{R}^{3}$, it can be extended analytically to the Poincaré sphere by projecting each point $\left(x_{1}, x_{2}, 1\right) \in \mathbb{R}^{3}$ onto the Poincaré sphere using a straight line through $\left(x_{1}, x_{2}, 1\right)$ 
PHASE PORTRAITS OF PIECEWISE LINEAR CONTINUOUS DIFFERENTIAL SYSTEMS 5

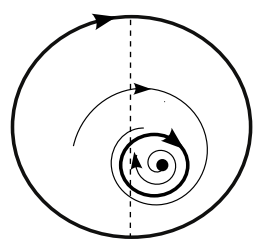

$2.1 S=3, R=2$

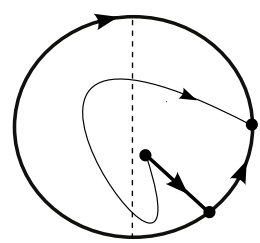

$2.4 S=6, R=1$

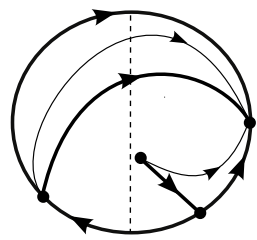

$2.7 S=9, R=2$

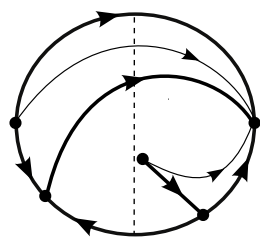

$2.10 S=11, R=2$

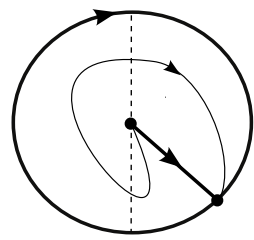

$2.13 S=4, R=1$

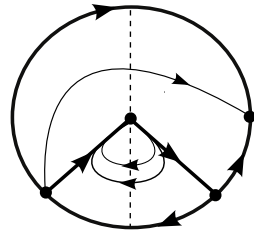

$2.16 S=9, R=2$

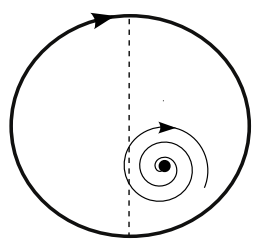

$2.2 S=2, R=1$

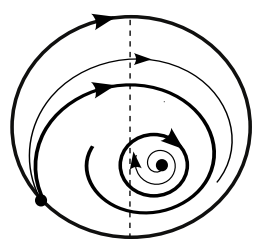

$2.5 S=5, R=2$

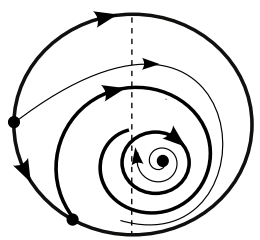

$2.8 S=6, R=2$

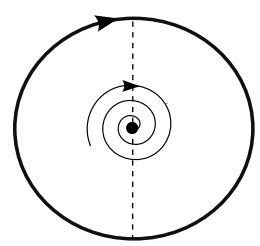

$2.11 S=2, R=1$

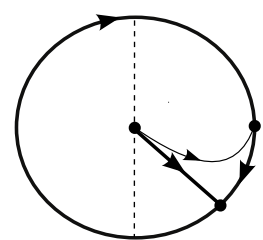

$2.14 S=6, R=1$

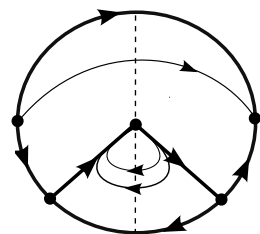

$2.17 S=11, R=2$

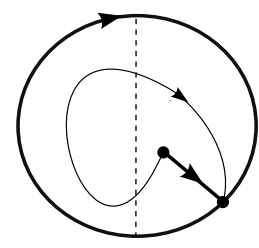

$2.3 S=4, R=1$
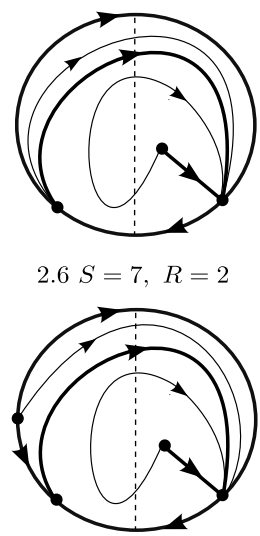

$2.9 S=9, R=2$

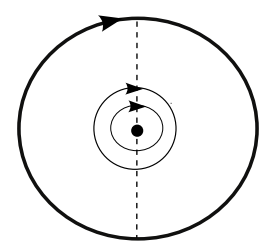

$2.12 S=2, R=1$

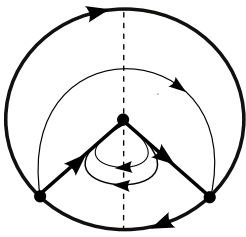

$2.15 S=7, R=2$

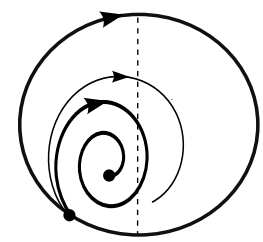

$2.18 S=4, R=1$

Figure 2. Topological phase portraits of system (3) with $k_{1}<$ $0, k_{2}>0$ in Theorem 2 . 
and the origin of $\mathbb{R}^{3}$. In this way we obtain a new differential system formed by two copies of (6): one on the northern hemisphere $\mathbb{S}^{-}=\left\{\left(s_{1}, s_{2}, s_{3}\right) \in \mathbb{S}^{2}, s_{3}>0\right\}$ and another on the southern hemisphere $\mathbb{S}^{+}=\left\{\left(s_{1}, s_{2}, s_{3}\right) \in \mathbb{S}^{2}, s_{3}<0\right\}$. Note that the equator $\mathbb{S}^{1}=\left\{\left(s_{1}, s_{2}, s_{3}\right) \in \mathbb{S}^{2}, s_{3}=0\right\}$ corresponds to the infinity of $\mathbb{R}^{2}$.

The local charts needed for doing the calculations on the Poincaré sphere are

$$
U_{i}=\left\{\mathbf{s} \in \mathbb{S}^{2}: s_{i}>0\right\}, V_{i}=\left\{\mathbf{s} \in \mathbb{S}^{2}: s_{i}<0\right\},
$$

where $\mathbf{s}=\left(s_{1}, s_{2}, s_{3}\right)$.

The expression for the corresponding differential system on $\mathbb{S}^{2}$ in the local chart $U_{1}$ is given by

$$
\dot{u}=v^{d}\left[-u P\left(\frac{1}{v}, \frac{u}{v}\right)+Q\left(\frac{1}{v}, \frac{u}{v}\right)\right], \quad \dot{v}=-v^{d+1} P\left(\frac{1}{v}, \frac{u}{v}\right)
$$

with $v>0$.

The expression for the corresponding differential system on $\mathbb{S}^{2}$ in the local chart $U_{2}$ is given by

$$
\dot{u}=v^{d}\left[-u P\left(\frac{u}{v}, \frac{1}{v}\right)-u Q\left(\frac{u}{v}, \frac{1}{v}\right)\right], \quad \dot{v}=-v^{d+1} Q\left(\frac{u}{v}, \frac{1}{v}\right)
$$

with $v>0$.

The expression for the corresponding differential system on $\mathbb{S}^{2}$ in the local chart $V_{j}$ is the same than in the chart $U_{j}$ multiplied by $(-1)^{d}$, for $j=1,2$.

The expression for the corresponding differential system on $\mathbb{S}^{2}$ in the local charts $U_{3}$ and $V_{3}$ are just

$$
\dot{u}=P(u, v), \dot{v}=Q(u, v)
$$

We note that to study the differential system (6), it is enough to study its Poincaré compactification restricted to the northern hemisphere plus $\mathbb{S}^{1}$. To draw the phase portraits we will consider the orthogonal projection $\pi\left(s_{1}, s_{2}, s_{3}\right)=\left(s_{1}, s_{2}\right)$ of the northern hemisphere onto the closed unit disc centered at the origin of coordinates in the plane $x_{3}=0$, called the Poincar disc.

Finite singular points of (6) are the singular points of its compactification which are in $\mathbb{S}^{2} \backslash \mathbb{S}^{1}$, and they can be studied using $U_{3}$. Infinite singular points of $(6)$ are the singular points of the corresponding differential system on the Poincaré disc lying on $\mathbb{S}^{1}$. Note that for studying the infinite singular points it suffices to look the ones at $\left.U_{1}\right|_{v=0},\left.V_{1}\right|_{v=0}$, and the origins of $U_{2}$ and $V_{2}$.

We note that the coordinates $(u, v)$ means different things in every local charts, but in the local charts $U_{i}$ and $V_{i}$ for $i=1,2$ the infinite points have always the coordinate $v=0$.

2.2. Chart $\mathbf{U}_{1}$. Let $x=\frac{1}{v}, y=\frac{u}{v}, v>0$, then system (3) becomes

$$
\begin{aligned}
& \frac{d u}{d t}=\varepsilon a v-\varepsilon-u^{2}+k_{1} u, \\
& \frac{d v}{d t}=v\left(k_{1}-u\right) .
\end{aligned}
$$


Let $v=0$, the infinite singular points of system (3) in chart $U_{1}$ should satisfy $u^{2}-k_{1} u+\varepsilon=0$. Recall that $\varepsilon>0$ and $\Delta_{1}=k_{1}^{2}-4 \varepsilon$, we have

Proposition 4. For system (11) the following statements hold.

(I) If $\Delta_{1}<0$, then system (11) has no infinite singular point.

(II) If $\Delta_{1}=0$, then system (11) has one infinite singular point $E=\left(\frac{k_{1}}{2}, 0\right)$, and it is a saddle-node, see Figure 3.

(III) If $\Delta_{1}>0$, then system (11) has two infinite singular points $E_{+}=\left(\frac{k_{1}+\sqrt{\Delta_{1}}}{2}, 0\right)$, which is a saddle for $k_{1}>0$ and a stable node for $k_{1}<0$. $E_{-}=$ $\left(\frac{k_{1}-\sqrt{\Delta_{1}}}{2}, 0\right)$, which is an unstable node for $k_{1}>0$, and a saddle for $k_{1}<0$.

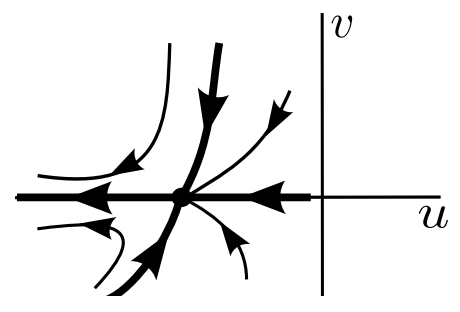

$3.1 k_{1}<0\left(k_{2}<0\right)$

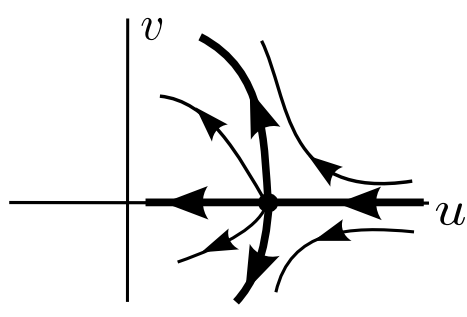

$3.2 k_{1}>0\left(k_{2}>0\right)$

Figure 3. The local phase portrait of the saddle-node singular point $E$ of system (11).

Proof. The singular points should satisfy the equation $u^{2}-k_{1} u+\varepsilon=0$. The statement (I) is obvious.

(II) Since $\varepsilon=\frac{k_{1}^{2}}{4}$, the Jordan matrix of singular point $E=\left(\frac{k_{1}}{2}, 0\right)$ is

$$
\left(\begin{array}{cc}
0 & \frac{k_{1}^{2} a}{4} \\
0 & \frac{k_{1}}{2}
\end{array}\right)
$$

We first consider the case $k_{1}<0$, we do the change $U=-\frac{k_{1}}{2}+u-\frac{k_{1}}{2} a v, V=$ $v, T=-t$, then system (11) becomes

$$
\begin{aligned}
& \frac{d U}{d T}=U^{2}+\frac{k_{1} a}{2} U V, \\
& \frac{d V}{d T}=V\left(-\frac{k_{1}}{2} k_{1}+U+\frac{k_{1} a}{2} V\right) .
\end{aligned}
$$

According to the Theorem 2.19 of the book [8], we can deduce that $E$ is a saddlenode singular point whose local phase portrait is described in Figure 3.1. Note that we have reverse the time, so the orientation of the orbits is the converse. 
The phase portraits of $E$ described in Figure 3.2 can be analyzed similarly for the case $k_{1}>0$.

(III) The Jacobian matrix for singular point $E_{+}=\left(\frac{k_{1}+\sqrt{\Delta_{1}}}{2}, 0\right)$ is

$$
\left(\begin{array}{cc}
-\sqrt{\Delta_{1}} & \varepsilon a \\
0 & \frac{k_{1}-\sqrt{\Delta_{1}}}{2}
\end{array}\right)
$$

If $k_{1}>0$, then $E_{+}$is a saddle. If $k_{1}<0$, then $E_{+}$is a stable node.

The Jacobian matrix for singular point $E_{-}=\left(\frac{k_{1}-\sqrt{\Delta_{1}}}{2}, 0\right)$ is

$$
\left(\begin{array}{cc}
\sqrt{\Delta_{1}} & \varepsilon a \\
0 & \frac{k_{1}+\sqrt{\Delta_{1}}}{2}
\end{array}\right)
$$

If $k_{1}>0$, then $E_{-}$is an unstable node. If $k_{1}<0$, then $E_{-}$is a saddle.

2.3. Chart $\mathbf{V}_{1}$. Let $x=\frac{1}{v}, y=\frac{u}{v}, v<0$, then system (3) becomes

$$
\begin{aligned}
& \frac{d u}{d t}=\varepsilon a v-\varepsilon-u^{2}+k_{2} u, \\
& \frac{d v}{d t}=v\left(k_{2}-u\right) .
\end{aligned}
$$

It is worth to note that system (16) is similar to (11), and just substitute $k_{1}$ of system (11) for $k_{2}$ of system (16). The proof of the next proposition uses the same arguments than the proof of Proposition 4.

Proposition 5. For system (16) the following statements hold.

(I) If $\Delta_{2}<0$, then system (16) has no infinite singular point.

(II) If $\Delta_{2}=0$, then system (16) has one infinite singular point $\tilde{E}=\left(\frac{k_{2}}{2}, 0\right)$, which is a saddle-node, see Figure 3.

(III) If $\Delta_{2}>0$, then system (16) has two infinite singular points $\tilde{E}_{+}=\left(\frac{k_{2}+\sqrt{\Delta_{2}}}{2}, 0\right)$, which is a saddle for $k_{2}>0$ and a stable node for $k_{2}<0$. $\tilde{E}_{-}=$ $\left(\frac{k_{2}-\sqrt{\Delta_{2}}}{2}, 0\right)$, which is an unstable node for $k_{2}>0$ and a saddle for
$k_{2}<0$.

2.4. Charts $\mathbf{U}_{2}$ and $V_{2}$. Let $x=\frac{u}{v}, y=\frac{1}{v}, v>0$, then system (3) becomes

$$
\begin{aligned}
& \frac{d u}{d t}=1-\varepsilon a u v+\varepsilon u^{2}-k_{1} u, \\
& \frac{d v}{d t}=\varepsilon v(u-a v) .
\end{aligned}
$$


with $u \geqslant 0$, and

$$
\begin{aligned}
& \frac{d u}{d t}=1-\varepsilon a u v+\varepsilon u^{2}-k_{1} u, \\
& \frac{d v}{d t}=\varepsilon v(u-a v),
\end{aligned}
$$

with $u \leqslant 0$.

Since $(0,0)$ is neither a singular point of system (17) nor a singular point of system (18), the origins of $U_{2}$ and $V_{2}$ are not infinite singular points.

2.5. Chart $\mathbf{U}_{3}$. It is obvious that system (3) has one finite singular point $(a, f(a))$. The local phase portrait of the singular point $(a, f(a))$ is characterized in the result.

Proposition 6. For system (3) with $k_{2}>0$ and $\varepsilon>0$ sufficiently small the following statements hold.

(I) If $a>0$ then the unique singular point is $\left(a, k_{1} a\right)$, which is unstable (stable) when $k_{1}<0\left(k_{1}>0\right)$. Furthermore,

(I.1) If $\Delta_{1}<0$, then $\left(a, k_{1} a\right)$ is a focus.

(I.2) If $\Delta_{1} \geqslant 0$, then $\left(a, k_{1} a\right)$ is a node.

(II) If $a=0$ then the unique singular point is $(0,0)$. Furthermore,

(II.1) If $\Delta_{1}<0, \Delta_{2}<0$ and $k_{1}<0, k_{1}+k_{2}<0$, then $(0,0)$ is a global unstable focus.

(II.2) If $\Delta_{1}<0, \Delta_{2}<0$ and $k_{1}<0, k_{1}+k_{2}=0$, then $(0,0)$ is a global center.

(II.3) If $\Delta_{1}<0, \Delta_{2}<0$ and $k_{1}<0, k_{1}+k_{2}>0$, then $(0,0)$ is a global stable focus.

(II.4) If $k_{1}<0$ and $\Delta_{1} \geqslant 0, \Delta_{2}<0$, then $(0,0)$ is an unstable node, see Figure 4.1.

(II.5) If $k_{1}<0$ and $\Delta_{1}<0, \Delta_{2} \geqslant 0$, then $(0,0)$ is a stable node, see Figure 4.2.

(II.6) If $k_{1}<0$ and $\Delta_{1} \geqslant 0, \Delta_{2} \geqslant 0$, then $(0,0)$ is formed by an elliptic sector and a hyperbolic sector, see Figure 4.3.

(II.7) If $k_{1}>0$ and $\Delta_{1}<0, \Delta_{2}<0$, then $(0,0)$ is a stable focus.

(II.8) If $k_{1}>0$, and either $\Delta_{1} \geqslant 0$ or $\Delta_{2} \geqslant 0$, then $(0,0)$ is a stable node, see Figure 4.4.

(III) If $a<0$ then the unique singular point $\left(a, k_{2} a\right)$ is stable. Furthermore,

(III.1) If $\Delta_{2}<0$, then $\left(a, k_{2} a\right)$ is a focus.

(III.2) If $\Delta_{2} \geqslant 0$, then $\left(a, k_{2} a\right)$ is a node.

We first introduce the results of Freire, Ponce and Torres [10], which is important for the proof of Proposition 6 .

Consider the following piecewise linear system

$$
\begin{aligned}
& \frac{d u}{d \tau}=F(u)-v, \\
& \frac{d v}{d \tau}=u-B v-A,
\end{aligned}
$$



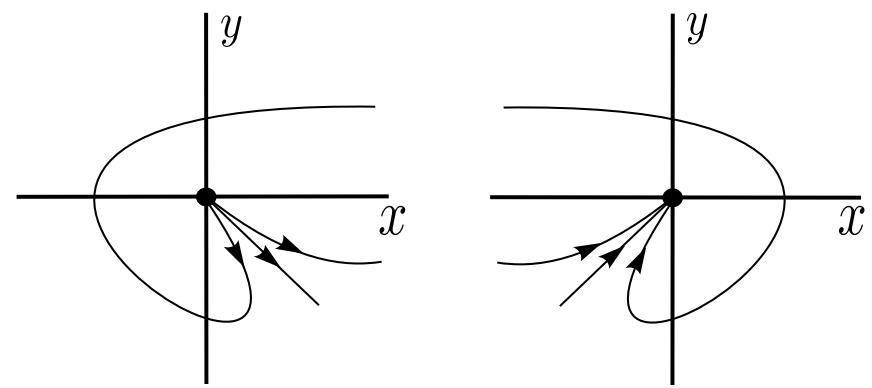

$4.1 k_{1}<0, k_{2}>0, \Delta_{1} \geq 0, \Delta_{2}<0 \quad 4.2 k_{1}<0, k_{2}>0, \Delta_{1}<0, \Delta_{2} \geq 0$
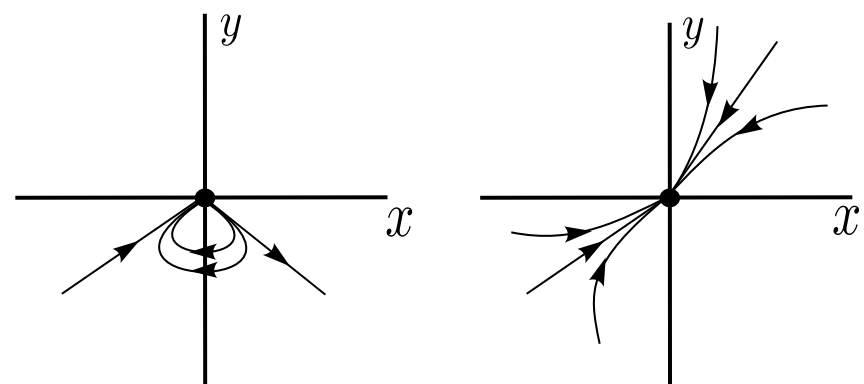

$4.3 k_{1}<0, k_{2}>0, \Delta_{1} \geq 0, \Delta_{2} \geq 0 \quad 4.4 k_{1}>0, k_{2}>0, \Delta_{1} \geq 0$, or $\Delta_{2} \geq 0$

FiguRE 4. The local phase portrait of the singular point $(a, f(a))$ of system (3) when $a=0$.

where

$$
F(u)= \begin{cases}\mu_{1} u & u>0, \\ \mu_{2} u & u \leqslant 0 .\end{cases}
$$

We introduce the following parameters which will simplify our analysis

$$
D_{i}=1-B \mu_{i}-\frac{\left(\mu_{i}-B\right)^{2}}{4}, \gamma_{i}=\frac{\mu_{i}-B}{2 \sqrt{D_{i}}}
$$

for $i=1,2$.

We have the following result, for a proof see Proposition 4 and Theorem 5 of $[10]$.

Lemma 7. The following statements hold for system (19) under the assumptions $D_{1}>0$ and $D_{2}>0$.

Assume $A=0$.

(i) If $\gamma_{1}+\gamma_{2}>0$ then $(0,0)$ is a global unstable nonlinear focus.

(ii) If $\gamma_{1}+\gamma_{2}=0$ then $(0,0)$ is a global nonlinear center.

(iii) If $\gamma_{1}+\gamma_{2}<0$ then $(0,0)$ is a global stable nonlinear focus.

Assume $A \neq 0$ and $\mu_{1} \mu_{2}<0$. 
PHASE PORTRAITS OF PIECEWISE LINEAR CONTINUOUS DIFFERENTIAL SYSTEMS 11

(iv) If $\gamma_{1}+\gamma_{2}>0$ and $\operatorname{sign}(A)=\operatorname{sign}\left(\mu_{2}\right)$, then the equilibrium point $(A, F(A))$ is asymptotically stable, and it is surrounded by a unique unstable limit cycle.

(v) If $\gamma_{1}+\gamma_{2} \leqslant 0$ and $\operatorname{sign}(A)=\operatorname{sign}\left(\mu_{2}\right)$, then the equilibrium point $(A, F(A))$ is global attractor and no limit cycle exist.

(vi) If $\gamma_{1}+\gamma_{2} \geqslant 0$ and $\operatorname{sign}(A)=\operatorname{sign}\left(\mu_{1}\right)$, then the equilibrium point $(A, F(A))$ is a global repeller and no limit cycle exist.

(vii) If $\gamma_{1}+\gamma_{2}<0$ and $\operatorname{sign}(A)=\operatorname{sign}\left(\mu_{1}\right)$, then the equilibrium point $(A, F(A))$ is unstable, and it is surrounded by a unique stable limit cycle.

Proof of Proposition 6. (I) The characteristic polynomial of system (3) at its equilibrium point is $\lambda^{2}+k_{1} \lambda+\varepsilon$. Recall that $\varepsilon>0$, then statements (I) follows easily.

(II) Let $u=\sqrt{\varepsilon} x, v=-y$ and $\tau=\sqrt{\varepsilon} t$, then system (3) can be written in the canonical form (19) with $A=\sqrt{\varepsilon} a, B=0$ and $\mu_{1}=-\frac{k_{1}}{\sqrt{\varepsilon}}, \mu_{2}=-\frac{k_{2}}{\sqrt{\varepsilon}}$. It is easy to compute that

$$
\begin{array}{rlrl}
D_{1} & =\frac{4 \varepsilon-k_{1}^{2}}{4 \varepsilon}, & \gamma_{1} & =-\frac{k_{1}}{\sqrt{4 \varepsilon-k_{1}^{2}}}, \\
D_{2}=\frac{4 \varepsilon-k_{2}^{2}}{4 \varepsilon}, & \gamma_{2}=-\frac{k_{2}}{\sqrt{4 \varepsilon-k_{2}^{2}}} .
\end{array}
$$

Recall that $a=0, k_{2}>0$ and $\varepsilon>0$ sufficiently small. If $\Delta_{1}<0$ and $\Delta_{2}<0$, then $D_{1}>0$ and $D_{2}>0$.

First we consider the case $\Delta_{1}<0, \Delta_{2}<0$. If $k_{1}<0, k_{2}>0$ and $k_{1}+k_{2}<0$, then $\gamma_{1}+\gamma_{2}>0$. From statement (i) of Lemma 7 , we can conclude that $(0,0)$ is an unstable focus. This is the proof of statement (II.1) of proposition 6. If $k_{1}<0, k_{2}<0$ and $k_{1}+k_{2}=0$, then $\gamma_{1}+\gamma_{2}=0$. From statement (ii) of Lemma $7,(0,0)$ is a global center. This is the proof of statement (II.2) of Proposition 6. If $k_{1}<0, k_{2}>0, k_{1}+k_{2}>0$ or $k_{1}>0, k_{2}>0$, then $\gamma_{1}+\gamma_{2}<0$. From statement (iii) of Lemma $7,(0,0)$ is a stable focus. This is the proof of statement (II.3) and (II.7) of Proposition 6.

In the following we discuss the other cases. Since the singular point $(0,0)$ is located in the switching line $x=0$ for $a=0$, the type and stability of $(0,0)$ depends on both differential systems, the one in $x>0$ and the other in $x<0$.

(II.4) For the case $k_{1}<0, k_{2}>0$ and $\Delta_{1} \geqslant 0, \Delta_{2}<0$, then $(0,0)$ is an unstable node in the right zone and a stable focus in the left zone, thus $(0,0)$ is an unstable focus, see Figure 4.1 .

(II.5) For the case $k_{1}<0, k_{2}>0$ and $\Delta_{1}<0, \Delta_{2} \geqslant 0$, then $(0,0)$ is an unstable focus in the right zone and a stable node in the left zone, thus $(0,0)$ is a stable node, see Figure 4.2.

(II.6) For the case $k_{1}<0, k_{2}>0$ and $\Delta_{1} \geqslant 0, \Delta_{2} \geqslant 0$, thus $(0,0)$ is formed by an elliptic sector and a hyperbolic sector, see Figure 4.3.

(II.8) For the case $k_{1}>0, k_{2}>0$ and either $\Delta_{1} \geqslant 0$ or $\Delta_{2} \geqslant 0$, then $(0,0)$ is a stable node, see Figure 4.4 . 
(III) The characteristic polynomial of systems (3) is $\lambda^{2}+k_{2} \lambda+\varepsilon$. Note that $\varepsilon>0$ and $k_{2}>0$, then statements (III) follows easily.

\section{Global phase portraits}

In this section we classify the global phase portraits of system (3) with $k_{1} k_{2} \neq 0$.

3.1. Global phase portraits of (3) with $k_{1}>0$ and $k_{2}>0$. First we consider the case $a>0$.

Proposition 8. The phase portrait of system (3) with $k_{1}>0, k_{2}>0$ and $a>0$ is topologically equivalent to

(I) Figure 1.1 if $\Delta_{1}<0$ and $\Delta_{2}<0$;

(II) Figure 1.2 if $\Delta_{1}=0$ and $\Delta_{2}<0$;

(III) Figure 1.3 if $\Delta_{1}>0$ and $\Delta_{2}<0$;

(IV) Figure 1.4 if $\Delta_{1}<0$ and $\Delta_{2}=0$;

(V) Figure 1.5 if $\Delta_{1}=0$ and $\Delta_{2}=0$;

(VI) Figure 1.6 if $\Delta_{1}>0$ and $\Delta_{2}=0$;

(VII) Figure 1.7 if $\Delta_{1}<0$ and $\Delta_{2}>0$;

(VIII) Figure 1.8 if $\Delta_{1}=0$ and $\Delta_{2}>0$;

(XI) Figure 1.9 if $\Delta_{1}>0$ and $\Delta_{2}>0$.

Proof. Recall that if $a>0, k_{1}>0$ and $k_{2}>0$, then system (3) has no limit cycles according to Proposition 2 of [10].

(I) For the case $\Delta_{1}<0$ and $\Delta_{2}<0$ the finite singular point $\left(a, k_{1} a\right)$ is a stable focus by statement (I.1) of Proposition 6, and by statement (I) of Propositions 4 and 5 the system has no infinite singular points. Therefore the global phase portrait is topologically equivalent to Figure 1.1.

(II) For the case $\Delta_{1}=0$ and $\Delta_{2}<0$ the finite singular point $\left(a, k_{1} a\right)$ is a stable node by statement (I.2) of Proposition 6 . The infinite singular point $E$ is a saddlenode by statement (II) of Proposition 4. Consequently the global phase portrait is topologically equivalent to Figure 1.2.

(III) For the case $\Delta_{1}>0$ and $\Delta_{2}<0$ the finite singular point $\left(a, k_{1} a\right)$ is a stable node by statement (I.2) of Proposition 6 . The infinite singular points $E_{+}$is a saddle and $E_{-}$is an unstable node by statement (III) of Proposition 4 . So the global phase portrait is topologically equivalent to Figure 1.3.

(IV) For the case $\Delta_{1}<0$ and $\Delta_{2}=0$ the finite singular point $\left(a, k_{1} a\right)$ is a stable focus by statement (I.1) of Proposition 6 . The infinite singular point $\tilde{E}$ is a saddle-node by statement (II) of Proposition 5. Hence the global phase portrait is topologically equivalent to Figure 1.4 .

(V) For the case $\Delta_{1}=0$ and $\Delta_{2}=0$ the finite singular point $\left(a, k_{1} a\right)$ is a stable node by statement (I.2) of Proposition 6 . The infinite singular points $E$ and $\tilde{E}$ are 
two saddle-nodes by statement (II) of Propositions 4 and 5. Then the global phase portrait is topologically equivalent to Figure 1.5 .

(VI) For the case $\Delta_{1}>0$ and $\Delta_{2}=0$ the finite singular point $\left(a, k_{1} a\right)$ is a stable node by statement (I.2) of Proposition 6. The infinite singular points $E_{+}$is a saddle and $E_{-}$is an unstable node by statement (III) of Proposition 4 , and the infinite singular point $\tilde{E}$ is a saddle-nodes by statement (II) of Proposition 5. Therefore the global phase portrait is topologically equivalent to Figure 1.6.

(VII) For the case $\Delta_{1}<0$ and $\Delta_{2}>0$ the finite singular point $\left(a, k_{1} a\right)$ is a stable focus by statement (I.1) of Proposition 6. The infinite singular point $\tilde{E}_{+}$is a saddle, and the other infinite singular point $\tilde{E}_{-}$is an unstable node by statement (III) of Proposition 5. So the global phase portrait is topologically equivalent to Figure 1.7.

(VIII) For the case $\Delta_{1}=0$ and $\Delta_{2}>0$ the finite singular point $\left(a, k_{1} a\right)$ is a stable node by statement (I.2) of Proposition 6 . The infinite singular point $E$ is a saddle-node by statement (II) of Proposition 4 . The infinite singular point $\tilde{E}_{+}$is a saddle, and the other infinite singular point $\tilde{E}_{-}$is an unstable node by statement (III) of Proposition 5. Hence the global phase portrait is topologically equivalent to Figure 1.8.

(IX) For the case $\Delta_{1}>0$ and $\Delta_{2}>0$ the finite singular point $\left(a, k_{1} a\right)$ is a stable node by statement (I.2) of Proposition 6. The infinite singular point $E_{+}$is a saddle and $E_{-}$is an unstable node by statement (III) of Proposition 4 . The infinite singular point $\tilde{E}_{+}$is a saddle and $\tilde{E}_{-}$is an unstable node by statement (III) of Proposition 5. Consequently the global phase portrait is topologically equivalent to Figure 1.9.

Second we consider the case $a=0$.

Proposition 9. The phase portraits of system (3) with $k_{1}>0, k_{2}>0$ and $a=0$ is topologically equivalent to

(I) Figure 1.10 if $\Delta_{1}<0$ and $\Delta_{2}<0$;

(II) Figure 1.11 if $\Delta_{1}=0$ and $\Delta_{2}<0$;

(III) Figure 1.12 if $\Delta_{1}>0$ and $\Delta_{2}<0$;

(IV) Figure 1.11 if $\Delta_{1}<0$ and $\Delta_{2}=0$;

$(V)$ Figure 1.13 if $\Delta_{1}=0$ and $\Delta_{2}=0$;

(VI) Figure 1.14 if $\Delta_{1}>0$ and $\Delta_{2}=0$;

(VII) Figure 1.12 if $\Delta_{1}<0$ and $\Delta_{2}>0$;

(VIII) Figure 1.14 if $\Delta_{1}=0$ and $\Delta_{2}>0$;

(IX) Figure 1.15 if $\Delta_{1}>0$ and $\Delta_{2}>0$.

Proof. Recall that $k_{1}>0$ and $k_{2}>0$ and $a=0$.

(I) For the case $\Delta_{1}<0$ and $\Delta_{2}<0$ the finite singular point $(0,0)$ is a stable focus by statement (II.7) of Proposition 6. There is no infinite singular points by 
statement (I) of Propositions 4 and 5. So the global phase portrait is topologically equivalent to Figure 1.10 .

(II) For the case $\Delta_{1}=0$ and $\Delta_{2}<0$ the finite singular point $(0,0)$ is a stable node by statement (II.8) of Proposition 6 . The infinite singular point $E$ is a saddlenode by statement (II) of Proposition 4. Therefore the global phase portrait is topologically equivalent to Figure 1.11 .

(III) For the case $\Delta_{1}>0$ and $\Delta_{2}<0$ the finite singular point $(0,0)$ is a stable node by statement (II.8) of Proposition 6 . The infinite singular point $E_{+}$is a saddle and $E_{-}$is an unstable node by statement (III) of Proposition 4 . Hence the global phase portrait is topologically equivalent to Figure 1.12.

(IV) For the case $\Delta_{1}<0$ and $\Delta_{2}=0$ the finite singular point $(0,0)$ is a stable node by statement (II.8) of Proposition 6 . The infinite singular point $\tilde{E}$ is a saddlenode by statement (II) of Proposition 5. Consequently the global phase portrait is topologically equivalent to Figure 1.11 by the homeomorphism $h:(x, y) \rightarrow$ $(-X,-Y)$.

(V) For the case $\Delta_{1}=0$ and $\Delta_{2}=0$ the finite singular point $(0,0)$ is a stable node by statement (II.8) of Proposition 6 . The infinite singular points $E$ and $\tilde{E}$ are saddle-nodes by statement (II) of Propositions 4 and 5 , respectively. Then the global phase portrait is topologically equivalent to Figure 1.13.

(VI) For the case $\Delta_{1}>0$ and $\Delta_{2}=0$ the finite singular point $(0,0)$ is a stable node by statement (II.8) of Proposition 6. The infinite singular point $E_{+}$is a saddle, $E_{-}$is an unstable node by statement (III) of Proposition 4 , and $\tilde{E}$ is a saddle-node by statement (II) of Proposition 5. So the global phase portrait is topologically equivalent to Figure 1.14.

(VII) For the case $\Delta_{1}<0$ and $\Delta_{2}>0$ the finite singular point $(0,0)$ is a stable node by statement (II.8) of Proposition 6 . The infinite singular point $\tilde{E}_{+}$is a saddle, and $E_{-}$is an unstable node by statement (III) of Proposition 5 . Therefore the global phase portrait is topologically equivalent to Figure 1.12.

(VIII) For the case $\Delta_{1}=0$ and $\Delta_{2}>0$ the finite singular point $(0,0)$ is a stable node by statement (II.8) of Proposition 6. The infinite singular point $E$ is a saddlenode by statement (II) of Proposition $4, \tilde{E}_{+}$is a saddle, and $\tilde{E}_{-}$is an unstable node by statement (III) of Proposition 5. Consequently the global phase portrait is topologically equivalent to Figure 1.14.

(IX) For the case $\Delta_{1}>0$ and $\Delta_{2}>0$ the finite singular point $(0,0)$ is a stable node by statement (II.8) of Proposition 6 . The infinite singular point $E_{+}$is a saddle, $E_{-}$is an unstable node by statement (III) of Proposition $4, \tilde{E}_{+}$is a saddle, and $\tilde{E}_{-}$is an unstable node by statement (III) of Proposition 5 . Hence the global phase portrait is topologically equivalent to Figure 1.15 .

Finally, we consider the case $a<0$.

Proposition 10. The phase portraits of system (3) with $k_{1}>0, k_{2}>0$ and $a<0$ is topologically equivalent to

(I) Figure 1.1 if $\Delta_{1}<0$ and $\Delta_{2}<0$; 
PHASE PORTRAITS OF PIECEWISE LINEAR CONTINUOUS DIFFERENTIAL SYSTEMS 15

(II) Figure 1.4 if $\Delta_{1}=0$ and $\Delta_{2}<0$;

(III) Figure 1.7 if $\Delta_{1}>0$ and $\Delta_{2}<0$;

(IV) Figure 1.2 if $\Delta_{1}<0$ and $\Delta_{2}=0$;

(V) Figure 1.5 if $\Delta_{1}=0$ and $\Delta_{2}=0$;

(VI) Figure 1.8 if $\Delta_{1}>0$ and $\Delta_{2}=0$;

(VII) Figure 1.3 if $\Delta_{1}<0$ and $\Delta_{2}>0$;

(VIII) Figure 1.8 if $\Delta_{1}=0$ and $\Delta_{2}>0$;

(XI) Figure 1.9 if $\Delta_{1}>0$ and $\Delta_{2}>0$.

Proof. The proof of Proposition 10 is similar to the one of Proposition 8, and we omit it here.

3.2. Global phase portraits of (3) with $k_{1}<0$ and $k_{2}>0$. First we consider the case $a>0$.

Proposition 11. The phase portraits of system (3) with $k_{1}<0, k_{2}>0$ and $a>0$ is topologically equivalent to

(I) Figure 2.1 if $\Delta_{1}<0, \Delta_{2}<0$ and $k_{1}+k_{2}>0$;

(II) Figure 2.2 if $\Delta_{1}<0, \Delta_{2}<0$ and $k_{1}+k_{2} \leqslant 0$;

(III) Figure 2.3 if $\Delta_{1}=0$ and $\Delta_{2}<0$;

(IV) Figure 2.4 if $\Delta_{1}>0$ and $\Delta_{2}<0$;

(V) Figure 2.5 if $\Delta_{1}<0$ and $\Delta_{2}=0$;

(VI) Figure 2.6 if $\Delta_{1}=0$ and $\Delta_{2}=0$;

(VII) Figure 2.7 if $\Delta_{1}>0$ and $\Delta_{2}=0$;

(VIII) Figure 2.8 if $\Delta_{1}<0$ and $\Delta_{2}>0$;

(IX) Figure 2.9 if $\Delta_{1}=0$ and $\Delta_{2}>0$;

(X) Figure 2.10 if $\Delta_{1}>0$ and $\Delta_{2}>0$.

Proof. Recall that $a>0, k_{1}<0$ and $k_{2}>0$. According to statement (a) of Proposition 1 and Lemma 1 of [23], if $k_{1} k_{2}<0$ then system has at most one limit cycle.

(I) For the case $\Delta_{1}<0, \Delta_{2}<0$ and $k_{1}+k_{2}>0$, we have $\mu_{1} \mu_{2}<0$, $D_{1}>0, D_{2}>0, \gamma_{1}+\gamma_{2}<0$ and $\operatorname{sign}(A)=\operatorname{sign}\left(\mu_{1}\right)$. By statement (vii) of Lemma 7 the finite singular point $\left(a, k_{1} a\right)$ is an unstable focus and there is a unique stable limit cycle surround it. It is obvious that system (3) has no infinite singular points by statement (I) of Propositions 4 and 5 . Then the global phase portrait is topologically equivalent to Figure 2.1 .

(II) For the case $\Delta_{1}<0, \Delta_{2}<0$ and $k_{1}+k_{2} \leqslant 0$, we have $\mu_{1} \mu_{2}<0, D_{1}>$ $0, D_{2}>0, \gamma_{1}+\gamma_{2} \geqslant 0$ and $\operatorname{sign}(A)=\operatorname{sign}\left(\mu_{1}\right)$. According to statement (vi) of Lemma 7 , the finite singular point $\left(a, k_{1} a\right)$ is an unstable focus and there is 
no limit cycles. It is obvious that system (3) has no infinite singular points by statement (I) of Propositions 4 and 5. So the global phase portrait is topologically equivalent to Figure 2.2.

(III) For the case $\Delta_{1}=0$ and $\Delta_{2}<0$, then the finite singular point $\left(a, k_{1} a\right)$ is an unstable node by (I.2) of Proposition 6, and the infinite singular point $E$ is a saddle-node by statement (II) of Proposition 4. Hence the global phase portrait is topologically equivalent to Figure 2.3 .

(IV) For the case $\Delta_{1}>0$ and $\Delta_{2}<0$, then the finite singular point $\left(a, k_{1} a\right)$ is an unstable node by (I.2) of Proposition 6 , and the infinite singular point $E_{+}$is a stable node, and $E_{-}$is a saddle by statement (III) of Proposition 4. Consequently the global phase portrait is topologically equivalent to Figure 2.4.

(V) For the case $\Delta_{1}<0$ and $\Delta_{2}=0$, then the finite singular point $\left(a, k_{1} a\right)$ is an unstable focus by (I.1) of Proposition 6 , and the infinite singular point $\tilde{E}$ is a saddle-node by statement (II) of Proposition 5. Therefore the global phase portrait is topologically equivalent to Figure 2.5.

(VI) For the case $\Delta_{1}=0$ and $\Delta_{2}=0$, then the finite singular point $\left(a, k_{1} a\right)$ is an unstable node by (I.2) of Proposition 6 , and the infinite singular points $E$ and $\tilde{E}$ are saddle-nodes by statement (II) of Propositions 4 and 5 . So the global phase portrait is topologically equivalent to Figure 2.6.

(VII) For the case $\Delta_{1}>0$ and $\Delta_{2}=0$, then the finite singular point $\left(a, k_{1} a\right)$ is an unstable node by (I.2) of Proposition 6 , and the infinite singular points $E_{+}$ is a stable node, $E_{-}$is a saddle by statement (III) of Proposition 4 , and $\tilde{E}$ is a saddle-node by statement (II) of Proposition 5. Therefore the global phase portrait is topologically equivalent to Figure 2.7.

(VIII) For the case $\Delta_{1}<0$ and $\Delta_{2}>0$, then the finite singular point $\left(a, k_{1} a\right)$ is an unstable focus by (I.1) of Proposition 6 , and the infinite singular point $\tilde{E}_{+}$is a saddle, and $\tilde{E}_{-}$is an unstable node by statement (III) of Proposition 5 . Hence the global phase portrait is topologically equivalent to Figure 2.8.

(IX) For the case $\Delta_{1}=0$ and $\Delta_{2}>0$, then the finite singular point $\left(a, k_{1} a\right)$ is an unstable node by (I.2) of Proposition 6 , and the infinite singular point $E$ is a saddle-node by statement (II) of Proposition $4, \tilde{E}_{+}$is a saddle, and $\tilde{E}_{-}$is an unstable node by statement (III) of Proposition 5. Consequently the global phase portrait is topologically equivalent to Figure 2.9 .

(X) For the case $\Delta_{1}>0$ and $\Delta_{2}>0$, then the finite singular point $\left(a, k_{1} a\right)$ is an unstable node by (I.2) of Proposition 6 , and the infinite singular point $E_{+}$is a stable node, $E_{-}$is a saddle by statement (III) of Proposition $4, \tilde{E}_{+}$is a saddle, and $\tilde{E}_{-}$is an unstable node by statement (III) of Proposition 5. Hence the global phase portrait is topologically equivalent to Figure 2.10.

Second we consider the case $a=0$.

Proposition 12. The phase portraits of system (3) with $k_{1}<0, k_{2}>0$ and $a=0$ is topologically equivalent to

(I) Figure 2.11 if $\Delta_{1}<0, \Delta_{2}<0$ and $k_{1}+k_{2} \neq 0$; 
(II) Figure 2.12 if $\Delta_{1}<0, \Delta_{2}<0$ and $k_{1}+k_{2}=0$;

(III) Figure 2.13 if $\Delta_{1}=0$ and $\Delta_{2}<0$;

(IV) Figure 1.14 if $\Delta_{1}>0$ and $\Delta_{2}<0$;

(V) Figure 2.13 if $\Delta_{1}<0$ and $\Delta_{2}=0$;

(VI) Figure 2.15 if $\Delta_{1}=0$ and $\Delta_{2}=0$;

(VII) Figure 2.16 if $\Delta_{1}>0$ and $\Delta_{2}=0$;

(VIII) Figure 2.14 if $\Delta_{1}<0$ and $\Delta_{2}>0$;

(IX) Figure 2.16 if $\Delta_{1}=0$ and $\Delta_{2}>0$;

(X) Figure 2.17 if $\Delta_{1}>0$ and $\Delta_{2}>0$.

Proof. Recall that $a=0, k_{1}<0$ and $k_{2}>0$.

(I) Assume $\Delta_{1}<0, \Delta_{2}<0$ and $k_{1}+k_{2} \neq 0$. If $k_{1}+k_{2}>0$, then the finite singular point $(0,0)$ is a global stable focus by statement (II.1) of Proposition 6 . If $k_{1}+k_{2}<0$, then the finite singular point $(0,0)$ is a global unstable focus by statement (II.3) of Proposition 6. It is obvious that system (3) has no infinite singular point by statement (I) of Propositions 4 and 5. Then the global phase portrait is topologically equivalent to Figure 2.11 .

(II) Assume $\Delta_{1}<0, \Delta_{2}<0$ and $k_{1}+k_{2}=0$. The finite singular point $(0,0)$ is a global center by statement (II.2) of Proposition 6. It is obvious that system (3) has no infinite singular points by statement (I) of Proposition 4 and 5 . Hence the global phase portrait is topologically equivalent to Figure 2.12.

(III) For the case $\Delta_{1}=0$ and $\Delta_{2}<0$ the finite singular point $(0,0)$ is an unstable node by statement (II.4) of Proposition 6 . The infinite singular point $E$ is a saddle-node by statement (II) of Proposition 4. Consequently the global phase portrait is topologically equivalent to Figure 2.13.

(IV) For the case $\Delta_{1}>0$ and $\Delta_{2}<0$ the finite singular point $(0,0)$ is an unstable node by statement (II.4) of Proposition 6 . The infinite singular point $E_{+}$is a stable node, and $E_{-}$is a saddle by statement (III) of Proposition 4. So the global phase portrait is topologically equivalent to Figure 2.14 .

(V) For the case $\Delta_{1}<0$ and $\Delta_{2}=0$ the finite singular point $(0,0)$ is a stable node by statement (II.5) of Proposition 6 . The infinite singular point $\tilde{E}$ is a saddlenode by statement (II) of Proposition 5. Therefore the global phase portrait is topologically equivalent to Figure 2.13 .

(VI) For the case $\Delta_{1}=0$ and $\Delta_{2}=0$ the finite singular point $(0,0)$ is formed by an elliptic sector and a hyperbolic sector by statement (II.6) of Proposition 6. The infinite singular points $E$ and $\tilde{E}$ are saddle-node by statement (II) of Propositions 4 and 5 . Consequently the global phase portrait is topologically equivalent to Figure 2.15 .

(VII) For the case $\Delta_{1}>0$ and $\Delta_{2}=0$ the finite singular point $(0,0)$ is formed by an elliptic sector and a hyperbolic sector by statement (II.6) of Proposition 6 . The infinite singular point $E_{+}$is a stable node, $E_{-}$is a saddle by statement (III) 
of Proposition 4, and $\tilde{E}$ is a saddle-node by statement (II) of Proposition 5. Hence the global phase portrait is topologically equivalent to Figure 2.16.

(VIII) For the case $\Delta_{1}<0$ and $\Delta_{2}>0$ the finite singular point $(0,0)$ is a stable node by statement (II.5) of Proposition 6. The infinite singular point $\tilde{E}_{+}$is a saddle, and $\tilde{E}_{-}$is an unstable node by statement (III) of Proposition 5. Therefore the global phase portrait is topologically equivalent to Figure 2.14.

(IX) For the case $\Delta_{1}=0$ and $\Delta_{2}>0$ the finite singular point $(0,0)$ is formed by an elliptic sector and a hyperbolic sector by statement (II.6) of Proposition 6. The infinite singular point $E$ is a saddle-node by statement (II) of Proposition $4, \tilde{E}_{+}$is a saddle, and $\tilde{E}_{-}$is an unstable node by statement (III) of Proposition 5 . So the global phase portrait is topologically equivalent to Figure 2.16.

(XI) For the case $\Delta_{1}>0$ and $\Delta_{2}>0$ the finite singular point $(0,0)$ is formed by an elliptic sector and a hyperbolic sector by statement (II.6) of Proposition 6 . The infinite singular point $E_{+}$is a stable node, $E_{-}$is a saddle by statement (III) of Proposition $4, \tilde{E}_{+}$is a saddle, and $\tilde{E}_{-}$is an unstable node by statement (III) of Proposition 5 . Hence the global phase portrait is topologically equivalent to Figure 2.17 .

Finally we consider the case $a<0$.

Proposition 13. The phase portraits of system (3) with $k_{1}>0, k_{2}>0$ and $a<0$ is topologically equivalent to

(I) Figure 2.2 if $\Delta_{1}<0, \Delta_{2}<0$ and $k_{1}+k_{2} \geqslant 0$;

(II) Figure 2.1 if $\Delta_{1}<0, \Delta_{2}<0$ and $k_{1}+k_{2}<0$;

(III) Figure 2.5 if $\Delta_{1}=0$ and $\Delta_{2}<0$;

(IV) Figure 2.8 if $\Delta_{1}>0$ and $\Delta_{2}<0$;

(V) Figure 2.18 if $\Delta_{1}<0$ and $\Delta_{2}=0$;

(VI) Figure 2.6 if $\Delta_{1}=0$ and $\Delta_{2}=0$;

(VII) Figure 2.9 if $\Delta_{1}>0$ and $\Delta_{2}=0$;

(VIII) Figure 2.4 if $\Delta_{1}<0$ and $\Delta_{2}>0$;

(IX) Figure 2.7 if $\Delta_{1}=0$ and $\Delta_{2}>0$;

(X) Figure 2.10 if $\Delta_{1}>0$ and $\Delta_{2}>0$.

Proof. Recall that $a<0, k_{1}<0$ and $k_{2}>0$.

(I) For the case $\Delta_{1}<0, \Delta_{2}<0$ and $k_{1}+k_{2} \geqslant 0$, we have $\mu_{1} \mu_{2}<0, D_{1}>0, D_{2}>$ $0, \gamma_{1}+\gamma_{2} \leqslant 0$ and $\operatorname{sign}(A)=\operatorname{sign}\left(\mu_{2}\right)$. By statement $(\mathrm{v})$ of Lemma 7 the finite singular point $\left(a, k_{2} a\right)$ is a stable focus and there is no limit cycles. It is obvious that system (3) has no infinite singular points by statement (I) of Propositions 4 and 5. Therefore the global phase portrait is topologically equivalent to Figure 2.2.

(II) For the case $\Delta_{1}<0, \Delta_{2}<0$ and $k_{1}+k_{2}<0$, we have $\mu_{1} \mu_{2}<0$, $D_{1}>0, D_{2}>0, \gamma_{1}+\gamma_{2}>0$ and $\operatorname{sign}(A)=\operatorname{sign}\left(\mu_{2}\right)$. Form statement (iv) of Lemma 7 the finite singular point $\left(a, k_{2} a\right)$ is a stable focus and there is a unique 
unstable limit cycle surround it. It is obvious that system (3) has no infinite singular points by statement (I) of Propositions 4 and 5 . Then the global phase portrait is topologically equivalent to Figure 2.1.

(III) For the case $\Delta_{1}=0$ and $\Delta_{2}<0$ the finite singular point $\left(a, k_{2} a\right)$ is a stable focus by (III.1) of Proposition 6. The infinite singular point $E$ is a saddle-node by statement (II) of Proposition 4. According to the Poincaré-Bendixson Theorem, there is at least one unstable limit cycle surround the singular point $\left(a, k_{2} a\right)$, and we know that when a limit cycle exists it is unique. Hence the global phase portrait is topologically equivalent to Figure 2.5.

(IV) For the case $\Delta_{1}>0$ and $\Delta_{2}<0$ the finite singular point $\left(a, k_{2} a\right)$ is a stable focus by (III.1) of Proposition 6. The infinite singular point $E_{+}$is a stable node, $E_{-}$is a saddle by statement (III) of Proposition 4. By the Poincaré-Bendixson Theorem there is a limit cycle surround the singular point $\left(a, k_{2} a\right)$, and we know that when a limit cycle exists is unique, and consequently unstable. So the global phase portrait is topologically equivalent to Figure 2.8 .

(V) For the case $\Delta_{1}<0$ and $\Delta_{2}=0$ the finite singular point $\left(a, k_{2} a\right)$ is a stable node by (III.2) of Proposition 6. The infinite singular point $\tilde{E}$ is a saddle-node by statement (II) of Proposition 5. So the global phase portrait is topologically equivalent to Figure 2.18.

(VI) For the case $\Delta_{1}=0$ and $\Delta_{2}=0$ the finite singular point $\left(a, k_{2} a\right)$ is a stable node by (III.2) of Proposition 6. The infinite singular points $E$ and $\tilde{E}$ are saddle-nodes by statement (II) of Propositions 4 and 5, respectively. Then the global phase portrait is topologically equivalent to Figure 2.6.

(VII) For the case $\Delta_{1}>0$ and $\Delta_{2}=0$ the finite singular point $\left(a, k_{2} a\right)$ is a stable node by (III.2) of Proposition 6 . The infinite singular point $E_{+}$is a stable node, $E_{-}$is a saddle by statement (III) of Proposition 4 , and $\tilde{E}$ is a saddle-node by statement (II) of Proposition 5. Therefore the global phase portrait is topologically equivalent to Figure 2.9.

(VIII) For the case $\Delta_{1}<0$ and $\Delta_{2}>0$ the finite singular point $\left(a, k_{2} a\right)$ is a stable node by (III.2) of Proposition 6 , and the infinite singular point $\tilde{E}_{+}$is a saddle, and $\tilde{E}_{-}$is an unstable node by statement (III) of Proposition 5 . Consequently the global phase portrait is topologically equivalent to Figure 2.4 .

(IX) For the case $\Delta_{1}=0$ and $\Delta_{2}>0$ the finite singular point $\left(a, k_{2} a\right)$ is a stable node by (III.2) of Proposition 6. The infinite singular points $E$ is a saddle-node by statement (II) of Proposition $4, \tilde{E}_{+}$is a saddle, and $\tilde{E}_{-}$is an unstable node by statement (III) of Proposition 5. Hence the global phase portrait is topologically equivalent to Figure 2.7.

(X) For the case $\Delta_{1}>0$ and $\Delta_{2}>0$ the finite singular point $\left(a, k_{2} a\right)$ is an unstable node by (III.2) of Proposition 6 . The infinite singular point $E_{+}$is a stable node, $E_{-}$is a saddle by statement (III) of Proposition $4, \tilde{E}_{+}$is a saddle, and $\tilde{E}_{-}$is an unstable node by statement (III) of Proposition 5. So the global phase portrait is topologically equivalent to Figure 2.10. 


\section{REMARKS}

In this paper we classify the global phase portraits for the class of piecewise linear continuous differential system (3) separated by the straight line $x=0$. We can find three main differences between the smooth and the piecewise smooth differential systems when we analyze the global phase portraits.

First, for the smooth differential systems the expressions for the charts $V_{i}, i=$ $1,2,3$ are those for the charts $U_{i}$ multiplied by $(-1)^{d-1}$, where $d$ is the degree of the polynomial differential systems. This symmetry property in general does not hold for piecewise smooth differential systems because the expressions for charts $V_{i}, i=1,2,3$ are different from the expressions of $U_{i}$ multiplied by $(-1)^{d-1}$, see for instance (11) and (16).

Second, for the smooth differential systems if $\mathbf{s} \in \mathbb{S}^{\mathbf{1}}$ is an infinite singular point, then $-\mathbf{s} \in \mathbb{S}^{\mathbf{1}}$ is another infinite singular point. Thus the number of infinite singular points is even and the local behaviour of one is that of the other multiplied by $(-1)^{d-1}$. But for the piecewise smooth differential systems in general the singular points at infinity are not diametrally opposite, see Figure 1.2 for example.

Finally, when a finite singular point of the piecewise smooth differential system is located at the separating line $x=0$, the analysis of its local phase portrait is more complex than in the smooth differential systems.

\section{Acknowledgements}

The first author is partially supported by the Natural Science Foundation of Guangdong Province (2017A030313010), National Natural Science Foundations of China (No. 11401111), China Scholarship Council (No. 201608440447) and Science and Technology Program of Guangzhou (No. 201707010426).

The second author is partially supported by the MINECO grants MTM201340998-P and MTM2016-77278-P (FEDER), the AGAUR grant 2014 SGR568, and the projecte MDM-2014-0445 (BGSMath).

\section{REFERENCES}

[1] Cao Y and Liu C 2017 The estimate of the amplitude of limit cycles of symmetric Liénard systems J. Differential Equations 262 2025-2038

[2] Chen H and Chen X 2015 Dynamical analysis of a cubic Liénard system with global parameters Nonlinearty 28 3535-3562

[3] Chen H and Chen X 2016 Dynamical analysis of a cubic Liénard system with global parameters (II) Nonlineart 29 1798-1826

[4] Coll B, Dumortier F and Prohens R 2013 Configurations of limit cycles in Liénard equations J. Differential Equations 255 4169-4184

[5] Desroches M, Freire E, Hogan S J, Ponce E and Thota P 2012 Canards in piecewise-linear systems: explosions and super-explosions Proceeding of the Royal Society A 4692012060318 $\mathrm{pp}$

[6] Desroches M, Guillamon A, Ponce E, Rodrigues E and Teruel A E 2016 Canards, folded nodes, and mixed-mode oscillations in piecewise-linear slow-fast systems SIAM Review 58 653-691

[7] Dumortier F 2006 Compactification and desigularization of spaces of polynomial Liénard equations J. Differential Equations 224 296-313 
[8] Dumortier F, Llibre J and Artés J C 2006 Qualitative Theory of Planar Differential Systems (Berlin Heidelberg: Springer-Verlag)

[9] Dumortier F, Panazzolo D and Roussarie R 2007 More limit cycles than expected in Liénard equations Proc. Amer. Math. Soc. 135 1895-1904

[10] Freire E, Ponce E and Torres F 1997 Hopf-like bifurcations in planar piecewise linear systems Publicacious Matematiques 41 135-148

[11] Freire E, Ponce E, Rodrigo F and Torres F 1998 Bifurcation sets of continuous piecewise linear systems with two zones, Internat. J. Bifur. Chaos 8 2073-2097

[12] Garcia F, Desroches M, Krupa M and Teruel A E 2015 Canard solutions in piecewise linear systems with three zones Dynamical Systems 31 173-197

[13] Gasull A and Hector G 2002 A new criterion for controlling the number of limit cycles of some generalized Liénard equations J. Differential Equations 185 54-73

[14] Gine J 2017 Liénard equation and its generalizations International Journal of Bifurcation and Chaos $2717500817 \mathrm{pp}$

[15] Gine J and Llibre J 2017 On the integrability of Liénard systems with a strong saddle Applied Mathematics Letters 70 39-45

[16] Han M, Tian Y and Yu P 2013 Small-amplitude limit cycles of polynomial Liénard systems Science China Mathematics 56 1543-1556

[17] Khibnik A I, Krauskopf B and Rousseau C 1998 Global study of cubic Liénard equations Nonlinearity 11 1505-1519

[18] Li C and Llibre J 2012 Uniqueness of limit cycles for Liénard differential equations of degree four J. Differential Equations 252 3142-3162

[19] Li C and Lu K 2014 Slow divergence integral and its application to classical Liénard equations of degree five J. Differential Equations 257 4437-4469

[20] Liénard A 1928 Étude des oscillations entrenues Revue Génerale de 1' Élilectricité 23 946-954

[21] Lins C, Melo W and Pugh C C 1977 On Liénards equation Lecture Notes in Math. 597 $335-357$

[22] Llibre J, Mereu A C and Teixeira M A 2010 Limit cycles of the generalized polynomial Liénard differential equations Math. Proc. Cambridge Philos. Soc. 148 363-383

[23] Llibre J, Ordoñez M and Ponce E 2013 On the existence and uniquess of limit cycles in planar continuous piecewise linear systems without symmetry Nonlinear Anal. Real World Appl. 14 2002-2012

[24] Llibre J, Ponce E and Valls C 2015 Uniqueness and non-uniqueness of limit cycles for piecewise linear differential systems with three zones and no symmetry J. Nonlinear Sci. 25 861-887

[25] Llibre J and Teixeira M A 2014 Limit cycles for $m$-piecewise discontinuous polynomial Liénard differential equations $Z$. Angew. Math. Phys. 66 51-66

[26] Llibre J and Valls C 2010 Liouvillian first integrals for Liénard Polynomial differential systems Proc. Amer. Math. Soc. 138 3229-3239

[27] Llibre J and Zhang X 2017 Limit cycles of the classical Liénard differential systems, A survey on the Lins Neto, de Melo and Pugh's conjecture Expo. Math. 35 286-299

[28] Lum R and Chua L.O. 1991 Global properties of continuous piecewise-linear vector fields. Part I: Simplest case in $\mathbb{R}^{2}$, Internat. J. Circuit Theory Appl. 19 251-307

[29] Lum R and Chua L.O. 1991 Global properties of continuous piecewise-linear vector fields. Part II: simplest symmetric in $\mathbb{R}^{2}$, Internat. J. Circuit Theory Appl. 20 9-46

[30] Maesschalck P De and Dumortier F 2011 Classical Liénard equation of degree $n=6$ or $n>6$ can have $[(n-1) / 2]+2$ limit cycles J. Differential Equations $2502162-2176$

[31] Maesschalck P De and Huzak R 2015 Slow divergence integrals in classical Liénard equations near centers J. Dynam. Differential Equations 27 177-185

[32] Martins M, Mereu R and Cristina A 2014 Limit cycles in discontinuous classical Liénard eqautions Nonlinear Anal. Real. World Appl. 20 67-73

[33] Prohens R, Teruel A E and Vich C 2016 Slow-fast n-dimensional piecewise linear differential systems J. Differential Equations 260 1865-1892

[34] Roberts A 2016 Canard Explosion and Relaxation Oscillation in Planar, Piecewise-Smooth, Continuous System SIAM. J. Applied Dyn. System 15 609-624

[35] Robert A and Gendinning P 2014 Canard-like phenomena in piecewise-smooth van der Pol systems Chaos $2402313811 \mathrm{pp}$ 
[36] Shen H and Han M 2013 Bifurcations of canard Limit cycles in several singularly perturbed Generalized polynomial Liénard systems Discrete and Continuous Dynamical Systems 33 3085-3108

[37] Xiong Y and Han M 2014 New lower bounds for the Hilbert number of polynomial systems of Liénard type J. Differential Equations 257 2565-2590

[38] Xu Q and Huang W 2011 The center conditions and local bifurcation of critical periods for a Liénard system Applied Mathematics and Computation 217 6637-6643

[39] Yang L and Zeng X 2013 The period function of Liénard systems Proceeding of the Royal Society of Edinburgh A 143 205-221

[40] Yang L and Zeng X 2015 An upper bound for the amplitude of limit cycles in Liénard systems with symmetry J. Differential Equations 258 2701-2710

[41] Yu Z and Zhang W 2016 Conditions for polynomial Liénard centers Science China Mathematics 59 411-424

1 School of Mathematics and Statistics, Guangdong University of Finance and Economics, Guangzhou, 510320, P.R. China

E-mail address: lism1983@126.com

2 Departament de Matemàtiques, Universitat Autònoma de Barcelona, 08193 Bellaterra, Barcelona, Catalonia, Spain

E-mail address: jllibre@mat.uab.cat 